\title{
An Application of HRI in Low-Cost Digital Manufacturing
}

\author{
Lavindra de Silva Gregory Hawkridge German Terrazas Marco Perez Hernandez \\ Alan Thorne Duncan McFarlane Yedige Tlegenov \\ Institute for Manufacturing (IfM), University of Cambridge, UK \\ lpd25@cam.ac.uk
}
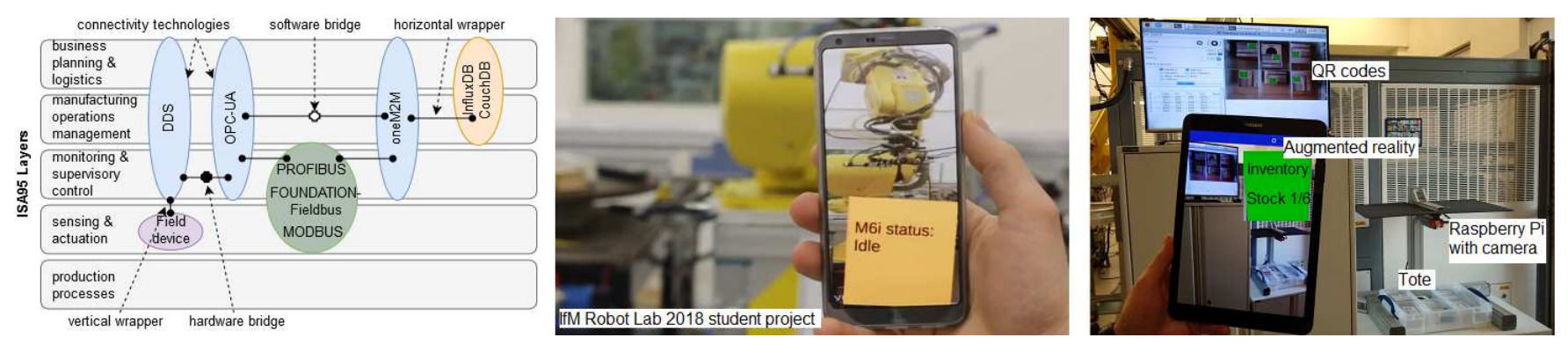

Figure 1: An instance of our SOA (left). Our robot monitoring (middle) and low-cost part tracking demonstrator (right).

\begin{abstract}
Digital Manufacturing (DM) broadly refers to applying digital information to enhance manufacturing processes, supply chains, products and services. In past work we proposed a low-cost DM architecture, supporting flexible integration of legacy robots. Here we discuss a demo of our architecture using an HRI scenario.
\end{abstract}

\section{KEYWORDS}

low-cost, digital manufacturing, services, legacy integration, SME

\section{MOTIVATION}

Our ultimate goal is the adoption of low-cost digital solutions by manufacturing SMEs, who need to keep development and legacy integration costs low and for solutions to be simple to deploy. To address these joint needs, we proposed in [1] the use of off-the-shelf and/or open source technologies, and a Service Oriented Architecture (SOA). A SOA is a framework for the composition of complex systems from autonomous, interoperable services, facilitating digitalisation and flexible integration of legacy machines and robots by "bolting on" low-cost technologies. A service represents devicespecific functionality, exposed via a well-defined interface. A service is realised by a connectivity technology $(\mathrm{CT})$, such as OPC-UA (OPC Unified Architecture) and DDS (Data Distribution Service) [2], which enables interoperable data and message exchange between heterogeneous technologies. A SOA requires certain core functionalities within a CT, such as addressing, discovery, service metadata exchange, and messaging.

Accepted to HRI 2020, The ACM/IEEE International Conference on Human-Robot Interaction, Cambridge, United Kingdom

(C) 2020 Copyright held by the owner/author(s).

This is the author's version of the work. It is posted here for your personal use. Not for redistribution. The definitive Version of Record was published in Companion of the 2020 ACM/IEEE International Conference on Human-Robot Interaction (HRI '20 Companion), March 23-26, 2020, Cambridge, United Kingdom, https://doi.org/10.1145/ 3371382.3378204 .
Two key features of our SOA for low-cost DM are wrapping legacy and newly added technologies as needed, and bridging CTs, as recommended by [2]. Wrapping may involve installing a CT on a robot, or on a Raspberry Pi attached to one, enabling the robot's discovery and incremental inclusion in the SOA. A bridge is a software module - possibly coupled with hardware - that performs protocol (data and message) translation between CTs. CTs may be bridged to connect parts of an SME's existing factory, e.g., if a robot only natively supports OPC-UA and a separate PLC network is already using DDS for data distribution. Figure 1 (left) depicts an instance of our architecture, comprising CTs (blue); low level industrial messaging protocols (green); and database technologies (orange), each wrapped in one oneM2M [2] CT. The instance can be reached incrementally, e.g. by (1) wrapping CouchDB in oneM2M, (2) wrapping the field device in a DDS service, (3) bridging DDS and OPC-UA, etc.

We will demonstrate aspects of our SOA: how a robot/machine and phone/tablet can each be wrapped in a CT, and automatically discover and use each other's services over Wifi. The tablet has an open source AR library, which we have configured to augment the robots/cells in a factory with status/condition information that humans can see quickly and intuitively from a safe distance. For portability, we will swap the robot cell with our low-cost cell that uses QR codes to identify parts and track their locations on shelves. The cell uses a Raspberry $\mathrm{Pi}$, a camera, and open source vision libraries; it supports instant scanning and storing of parts held in a tote by a human/robot, and sends stock levels to the tablet (Figure 1 middle and right).

\section{REFERENCES}

[1] G. Hawkridge, M. Perez Hernandez, L. de Silva, G. Terrazas, Y. Teglenov, D. Mcfarlane, and A. Thorne. 2019. Tying Together Solutions for Digital Manufacturing: Assessment of Connectivity Technologies \& Approaches. In Proceedings of the 24th IEEE ETFA. 1383-1387.

[2] R. Joshi, P. Didier, J. Jimenez, and T. Carey. 2017. The Industrial Internet of Things Volume G5: Connectivity Framework. Technical Report. Industrial Internet Consortium Report. 